\title{
Transcavernous Approach for Gross Total Resection of a Dumbbell-Shaped Giant Trigeminal Schwannoma
}

\author{
Sima Sayyahmelli ${ }^{1} \quad$ Emel Avci $^{1} \quad$ Burak Ozaydin $^{1}$ Mustafa K. Başkaya ${ }^{1}$ \\ ${ }^{1}$ Department of Neurological Surgery, University of Wisconsin School \\ of Medicine and Public Health, Madison, Wisconsin, United States \\ Address for correspondence Mustafa K. Başkaya, MD, Department of \\ Neurological Surgery, University of Wisconsin School of Medicine and \\ Public Health, Madison, Wisconsin, United States \\ J Neurol Surg B Skull Base 2022;83(suppl S3):e648-e649. \\ (e-mail: m.baskaya@neurosurgery.wisc.edu).
}

\begin{abstract}
Keywords

- dumbbell-shaped

- extradural transcavernous approach

- skull base

- transpetrosal transtentorial approach

- trigeminal schwannoma

Trigeminal schwannomas are rare nerve sheet tumors that represent the second most common intracranial site of occurrence after vestibular nerve origins. Microsurgical resection of giant dumbbell-shaped trigeminal schwannomas often requires complex skull base approaches. The extradural transcavernous approach is effective for the resection of these giant tumors involving the cavernous sinus.

The patient is a 72-year-old man with headache, dizziness, imbalance, and cognitive decline. Neurological examination revealed left-sided sixth nerve palsy, a diminished corneal reflex, and wasting of temporalis muscle. Magnetic resonance imaging (MRI) showed a giant homogeneously enhancing dumbbell-shaped extra-axial mass centered within the left cavernous sinus, Meckel's cave, and the petrous apex, with extension to the cerebellopontine angle. There was a significant mass effect on the brain stem causing hydrocephalus. Computed tomography (CT) scan showed erosion of the petrous apex resulting in partial anterior autopetrosectomy (-Figs. 1 and 2).

The decision was made to proceed with tumor resection using a transcavernous approach. Gross total resection was achieved. The surgery and postoperative course were uneventful, and the patient woke up the same as in the preoperative period. MRI confirmed gross total resection of the tumor. The histopathology was a trigeminal schwannoma, World Health Organization (WHO) grade I. The patient continues to do well without any recurrence at 15 -month follow-up.

This video demonstrates important steps of the microsurgical skull base techniques for resection of these challenging tumors.

The link to the video can be found at https://youtu.be/TMK5363836M
\end{abstract}

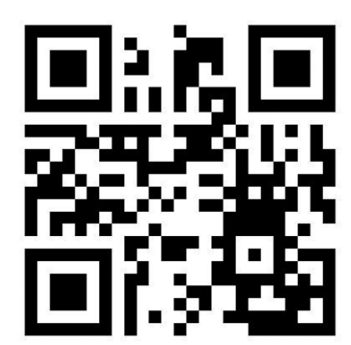

received

April 29, 2020

accepted after revision

January 9, 2021

published online

May 17, 2021
Conflict of Interest

None declared.

www.thieme.com/skullbasevideos

www.thieme.com/jnlsbvideos
Dol https://doi.org/ 10.1055/s-0041-1729995. ISSN 2193-6331.

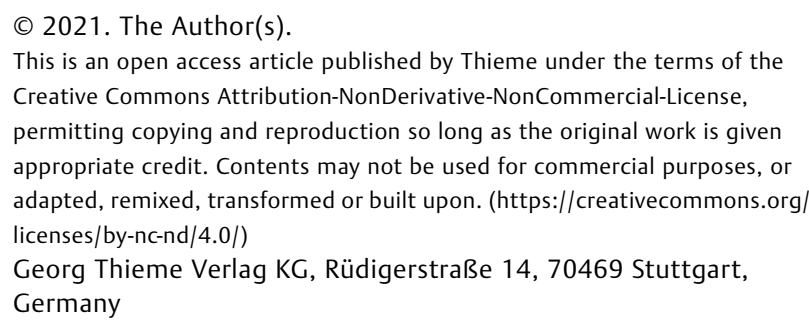




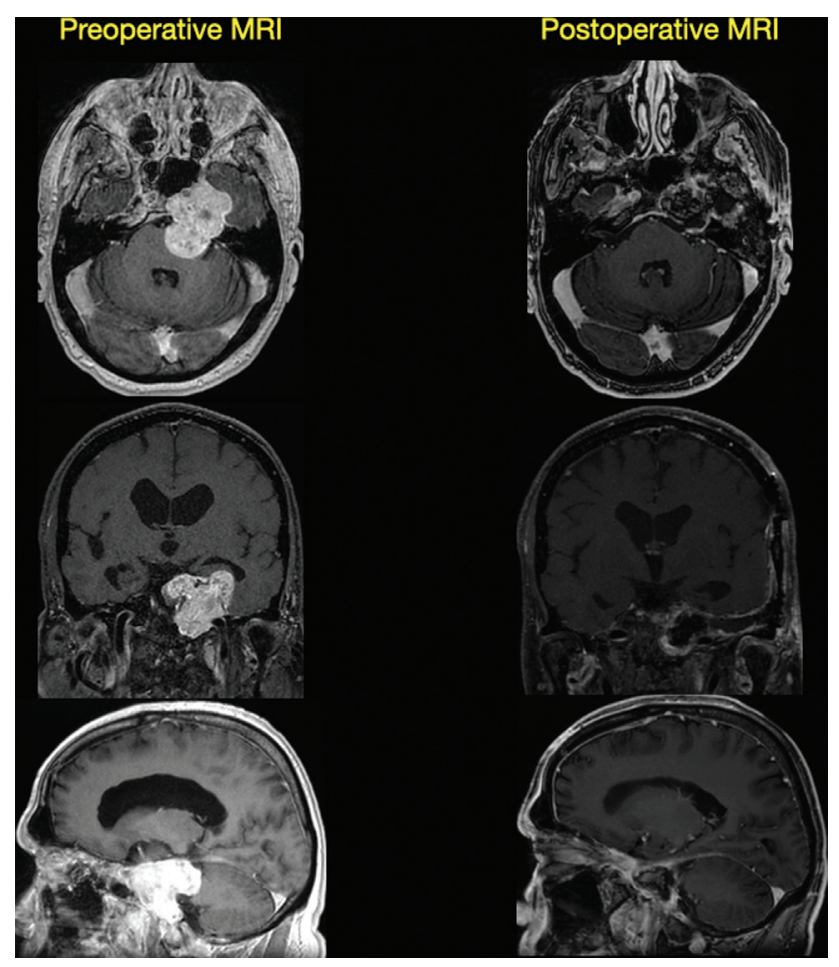

Fig. 1 Preoperative and postoperative MRI. MRI, magnetic resonance imaging.
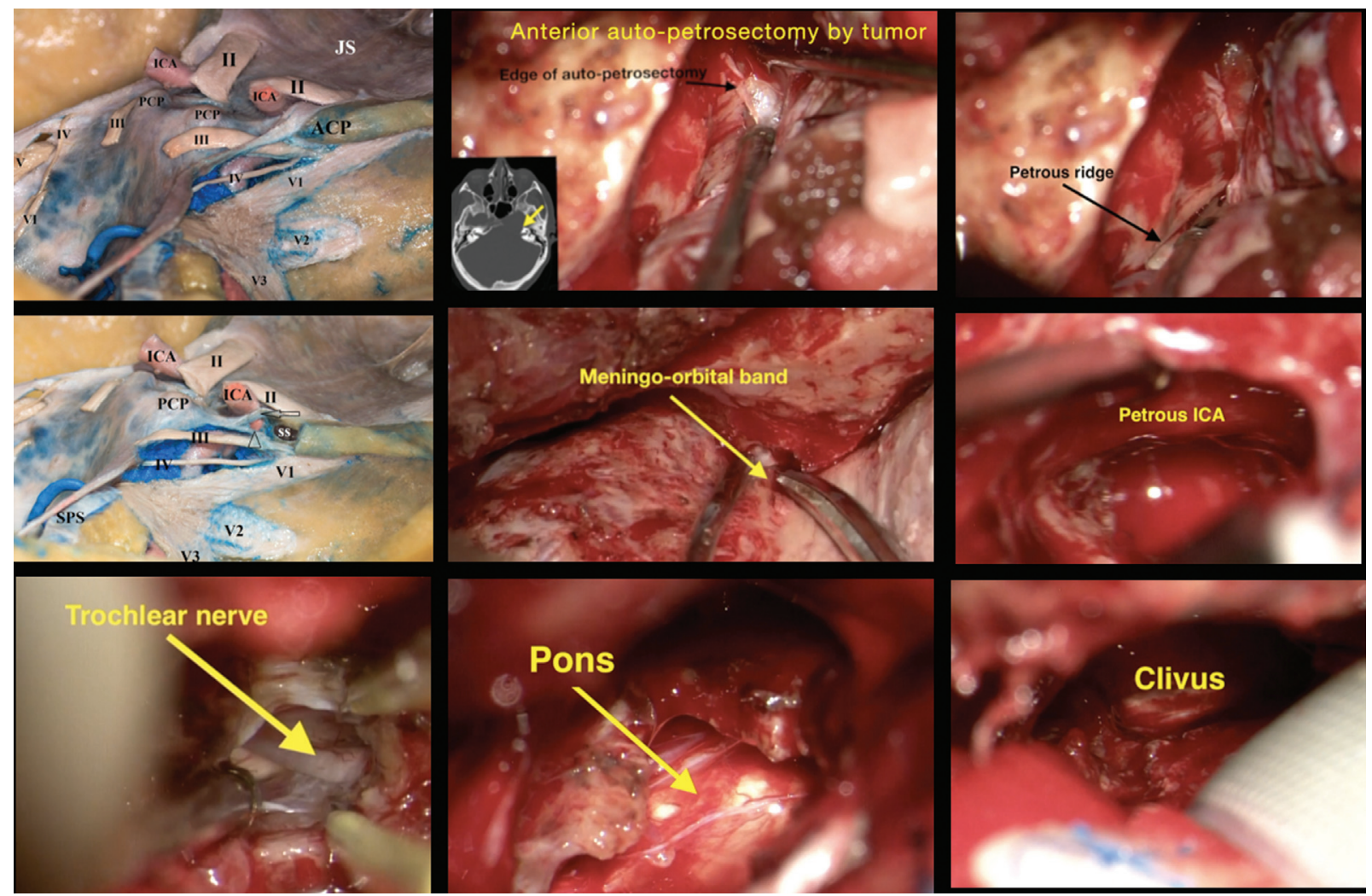

Fig. 2 Intraoperative images. ACP, anterior clinoid process; ICA, internal carotid artery; II, optic nerve; III, oculomotor nerve; IV, trochlear nerve; PCP, posterior clinoid process; SPS, superior petrosal sinus; V, trigeminal nerve; V1, ophthalmic division of trigeminal nerve; V2, maxillary division of trigeminal nerve; $\mathrm{V} 3$, mandibular division of trigeminal nerve; $\mathrm{VI}$, abducens nerve. 